\title{
Intelligent control system of seed cotton pretreatment based on knowledge base
}

\author{
Haopeng Wang ${ }^{1,2, a}$, Hui $\mathrm{Li}^{2, b^{,}{ }^{*}}$, Xianying Feng ${ }^{2, \mathrm{c}}$ \\ ${ }^{1}$ Shandong Police College, Jinan 250014, China \\ ${ }^{2}$ Key Laboratory of High Efficiency and Clean Mechanical Manufacture, Ministry of Education, \\ Shandong University, Jinan 250061, China \\ awhp-whp-whp@163.com, blihuifs@sdu.edu.cn, ${ }^{\mathrm{c}}$ fxying@ sdu.edu.cn \\ Corresponding Author: Li Hui
}

Keywords: seed cotton pretreatment; intelligent control; knowledge base; object oriented

\begin{abstract}
In order to improve the automation level of our country cotton processing and reduce reliance on operators production experience, this paper presented the seed cotton pretreatment of intelligent control strategy based on knowledge base and constructed the related control system. The system can detect the seed cotton characteristic which influenced the quality of seed cotton pretreatment online. Using the method of object oriented, the system represented the knowledge of seed cotton characteristic, device parameter and seed cotton grade standard. All the knowledge were stored into the knowledge base. The inference engine was the core of this system, it was a kind of hybrid inference mechanism based on the instance and the model. Validation test results showed that the system can formulate reasonable seed cotton pretreatment program according to the users requirements and the on line detected results of seed cotton characteristic. The system provided a basis for grading fine processing of cotton.
\end{abstract}

\section{Introduction}

At present, China's cotton processing level is relatively backward, seed cotton pretreatment before operating workers only with production experience or simple testing equipment to test the physical properties such as seed cotton moisture, impurities, and set the key parameter of the pretreatment equipment according to the test results, once the pre-treatment process began, cannot adjust according to the seed cotton real-time character of pretreatment equipment key parameters. To solve these problems, this paper constructs intelligent control system of seed cotton pretreatment based on knowledge base. The system can influence the on-line detection pretreatment of seed cotton quality traits, and according to the test results on the key operational parameters which can adaptively adjust the key operation parameters.

\section{Materials and methods}

\subsection{Characteristics analysis of seed cotton pretreatment.}

After pretreated, seed cotton should meet the following requirements:

1) Seed cotton moisture regains control between $6.5 \sim 8.5 \%$; 2) Break up a large group of seed cotton and make it fluffy, in the broken process, try to minimize damage to the cotton fiber and cotton seed, as well as impurities broken; 3) To clean up the impurity in the seed cotton as much as possible.

In order to make the seed cotton pretreated to meet the above requirements, cotton processing enterprises are mostly set in the seed preprocessing two drying process and two clean-up process.Before the actual processing, operator manual sampling of seed cotton physical properties.

In order to change the backward way of processing, the realization of intelligent adaptive control seed cotton pretreatment processing, need two important transformation to the existing production line: one is the on-line detection is added in the production line equipment, real-time detection of seed cotton each related physical properties; the second is building intelligent control knowledge base, 
real-time detection to the physics properties of seed cotton input knowledge base, through the study of intelligent reasoning, generate seed cotton pretreatment control scheme.

\subsection{Seed cotton pretreatment intelligent control system structure.}

According to the above the analysis of the characteristics of the seed cotton pretreatment process, in order to realize the intelligent control, need to seed cotton dry and clean up the implementation of each link process real-time selection and the key operation parameters real-time adjustment. The structure of the intelligent control system is shown in Fig.1.

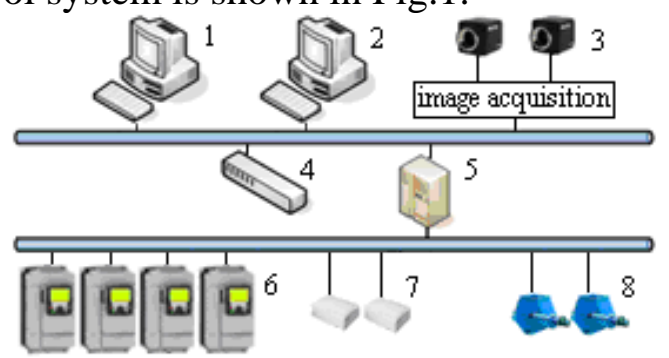

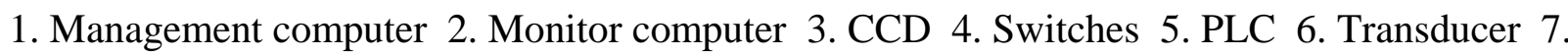
Temperature and humidity sensor 8 . Three-way valve

Fig. 1 Structure diagram of seed cotton pretreatment intelligent control system

\subsection{The representation of knowledge.}

The knowledge representation means using a computer to recognize the symbols or language description of objective things in the world. Using object-oriented representation method[1-3]. In the representation method of object-oriented, the things are known as the object. Characteristics of things are called attributes. The attribute information of seed cotton and conditioning regimen as shown in Table 1.

Table 1 the target of the system

\begin{tabular}{cc}
\hline Object Name & Property name \\
\hline Seed cotton & fiber length, fiber strength, moisture regain, the impurity rate, gloss yellowness \\
\hline $\begin{array}{c}\text { Pretreatment } \\
\text { scheme }\end{array}$ & $\begin{array}{c}\text { secondary drying whether or not to use, the secondary seed clear whether or not to use, a } \\
\text { level of drying air temperature, secondary drying air temperature, a tilt seed cleaner speed, } \\
\text { seed cleaner mention net speed, the secondary tilt seed cleaning machine speed, recycling } \\
\text { seed cleaning machine speed }\end{array}$ \\
\hline
\end{tabular}

In Table 1, lists the pretreatment process can affect the properties of seed cotton. Listed above is a description of the physical object, belongs to descriptive knowledge [4]. There are inferential knowledge and procedural knowledge. Inferential knowledge is mainly seed cotton properties and previous experience. Process knowledge is mainly dynamic knowledge model and algorithms.

\subsection{The composition of the knowledge base.}

The knowledge base of this system is designed based on relational database, divided into two groups: the fact that knowledge base and rule knowledge base. Among them, facts Knowledge includes three libraries, respectively is seed traits library, device library and cotton grade evaluation database; rules Knowledge Base includes a library is a pretreatment program library. The library of specific storage content is: 1) The seed cotton character library: Storage is testing before and after the pretreatment of seed cotton related traits.2) Device Library: Storage is the relevant information for the pre-treatment units.3) Evaluation grade cotton libraries: Storage is to rolling seed cottonevaluation standard of quality. 4) Conditioning regimen libraries: Storage is the seed cotton processing pretreatment scheme.

\subsection{Reasoning machine.}

Reasoning machine[5]is the thinking of this system, also is the core of system, the task is based on the actual detected seed cotton traits targeted pretreatment plan formulation. The system used is based on hybrid reasoning mechanism examples and models. When the reasoning based on the detected first seed traits search pretreatment program library, if preprocessing scheme in the library have suitable plan enter the pretreatment, if you will not find seed traits, knowledge generation device information into the model to calculate the new conditioning regimen, a new conditioning regimen 
deposited conditioning regimen library and output.This system development design of seed cotton dry and clean up the related experiments, through a large number of experimental data to set up seed cotton quality index model and cleaning equipment. Such as seed cotton drying model:

$\ln \left(M_{t} / M_{0}\right)=\ln \left(0.004056+0.001778 m_{1}+0.000762 m_{2}+0.001807 m_{3}\right)$

$-\left(0.048846+0.013957 m_{1}-0.013666 m_{2}+0.010769 m_{3}\right) t$

In the formula, when the $M_{\mathrm{t}}$ is $t$, carved material dry basis moisture content, \%; $M_{0}$ represents initial dry material moisture content, \%; $m_{1}$ represents feed quantity, $\mathrm{kg} / \mathrm{s} ; m_{2}$ represents the initial seed moisture content on a dry basis, $\% ; m_{3}$ represents air temperature, ${ }^{\circ} \mathrm{C}$.

Seed cotton cleaning up model:

$$
\begin{aligned}
& y_{b s}=0.125136 x_{1}+0.973904 x_{2}+0.142767 x_{2}{ }^{2}+0.096358 x_{3}+0.043334 x_{4} \\
& y_{c b}=0.532746 x_{1}+0.195791 x_{1}{ }^{2}+0.512548 x_{2}+0.189375 x_{2}{ }^{2}+0.331857 x_{3}+0.131977 x_{3}{ }^{2} \\
& +0.456321 x_{4}+0.091552 x_{4}{ }^{2}+0.072580 x_{1} x_{4}+0.069607 x_{2} x_{4} \\
& y_{l c}=0.207676 x_{1}+0.969954 x_{3}+0.076258 x_{4} \\
& y_{d m}=0.167919 x_{1}+0.085373 x_{1}{ }^{2}+0.071168 x_{2}+0.904932 x_{3}+0.355524 x_{3}{ }^{2}+0.047911 x_{1} x_{3} \\
& y_{d c}=0.263682 x_{1}+0.053153 x_{1}{ }^{2}+0.937052 x_{3}+0.198417 x_{3}{ }^{2} \\
& y_{b c}=0.202971 x_{1}+0.056683 x_{1}{ }^{2}+0.937028 x_{3}+0.210901 x_{3}{ }^{2}+0.158377 x_{4} \\
& y_{p p}=0.168519 x_{1}+0.807108 x_{3}+0.360438 x_{3}{ }^{2} \\
& y_{t r}=0.206620 x_{1}+0.818851 x_{3}+0.202347 x_{4}
\end{aligned}
$$

In the formula, $y_{\mathrm{bs}}, y_{\mathrm{cb}}, y_{\mathrm{lc}}, y_{\mathrm{dm}}, y_{\mathrm{dc}}, y_{\mathrm{bc}}, y_{\mathrm{pp}}$ and $y_{\mathrm{tr}}$ respectively represents boll shell, cotton sticks, leaf litter, dust mixed, stiff flap, sterile seeds, plastic bags and other debris and lint eight kinds of impurities cleaning efficiency, \%; $x_{1}, x_{2}, x_{3}$ and $x_{4}$ respectively represents a level tilt seed cleaning machine barbed nail drum rotational speed, toothed roller speed net seed cleaning machine, secondary tilt seed cleaning machine barbed nail roller speed and recovery of seed cleaning machine barbed nail roller speed and $\mathrm{r} / \mathrm{min}$.

\subsection{User requirements of seed cotton quality evaluation.}

Evaluation of seed quality is affected by multiple indicators. When solving multi-objective optimization, we often first transformed into single objective optimization to solve. This system from the perspective of user needs, constructed evaluation function:

$$
V(f(x))=\sum_{i=1}^{l} \lambda_{i} f_{i}(x)
$$

In the formula, $f_{\mathrm{i}}(x)$ is the $i$-th optimization index, in order to eliminate different index order of magnitude difference influence on the final result, you need to do all the optimization index normalized, all $f(x)$ are normalized to optimize treatment of the latter indicators; $\lambda_{i}$ is the $i$-th optimization index weights, it represents a relatively important objective $f_{\mathrm{i}}(x)$ of degree.

\section{Results and discussion}

To test the control effect of this system, contrast tests are carried out. Design of experiment is as follows: processing for 48 hours in a row, including 24 hours before using this control system, the equipment operation parameters fixed (two-stage drying tower hot blast temperature is $100{ }^{\circ} \mathrm{C}$, primary tilt seed cleaning machine, secondary tilt seed cleaning machine and recycling seed cleaning machine speed of $500 \mathrm{r} / \mathrm{min}$, defecation seed cleaning machine speed $1000 \mathrm{r} / \mathrm{min}$ ). 24 hours after the use of the control system, control strategy is to improve the cleaning efficiency (including the importance of the impurity rate of $50 \%$, after cleaning up the impurity rate shall not exceed $3.5 \%$, the importance of the fiber damage index was $30 \%$, and cleaning up after the length of fiber damage shall not exceed 3\%, 20\%) to the importance of the other indicators, test data as shown in Fig.3(data for all the testing data of the average 1 hour). 


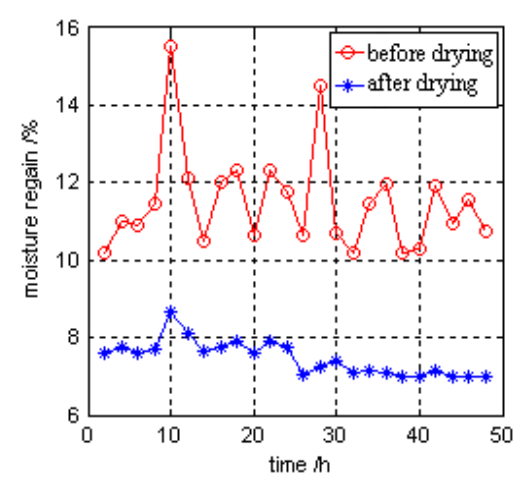

a. Curves of moisture regain

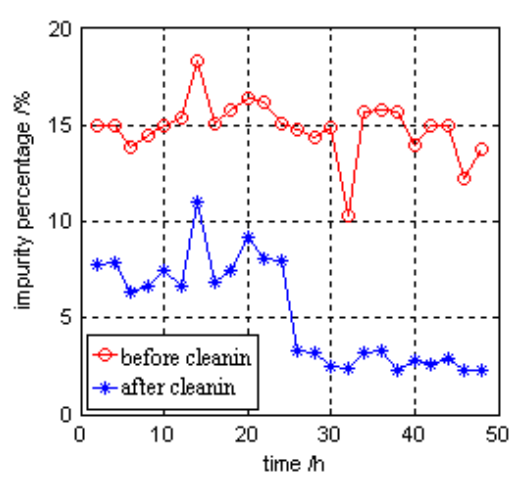

b. Curves of impurity content

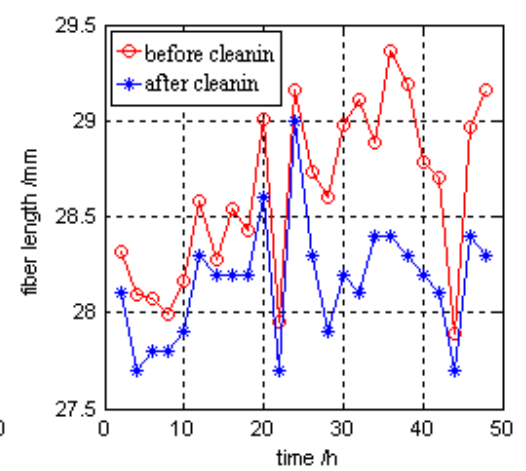

c. Curves of fibre length

Fig. 2 Curves of moisture regain impurity content and fibre length

As you can see from Fig.2a, before drying moisture regain volatility in 24 hours before and after two more similar, but after drying the moisture regain of volatile within 24 hours before, poor consistency. After using this system to control the moisture regain fluctuations decreased significantly after 24 hours to dry, good consistency.Can be seen from the Fig.2b, after 24 hours before cleaning impurity rate is higher; within 24 hours, due to the use of the control strategy to improve the cleaning efficiency, trash content significantly decreased, the basic control between 2-3\%.As can be seen from Fig.2c, within the first 24 hours, the fiber length before and after cleaning up little change in the basic no more than 1.5\%; within 24 hours, the fiber length before and after cleaning up large changes substantially 1.6 to $3 \%$, this shows that the fiber damage. This is because the system is to improve the cleaning efficiency of the control strategy. Cleaning efficiency and fiber damage itself is a pair of opposed targets. Need to increase the speed to improve the cleaning efficiency of cleaning equipment, which led to the fibers more likely to be interrupted, so the fiber length becomes shorter.

\section{Conclusion}

The seed cotton pretreatment intelligent control system developed by this paper has been applied to three cotton processing enterprise, after two years of practice test for the user's high praise, achieved a seed pretreatment "because flowers with cars "and fine cotton grading and processing.

\section{References}

[1]. Wang Lei, Cai Wanjing, Li Yongli, et al. Under standing user's searching purpose by the object-oriented semantic concept knowledge base. Journal of Computational Information Systems. Vol. 6 (2010) No. 1, p. 221-227.

[2]. Chou Ihsin, Fan Chinfeng. An object-orented security knowledge framework for the nuclear safety system project. International Journal of Software Engineering and Knowledge Engineering. Vol. 20 (2010) No. 3, p. 347-365.

[3]. He Bin, Deng Zhongqiang, Lv Haifeng. Object-oriented knowledge modeling for conceptual design of mechanisms. International Journal of Database Theory and Application. Vol. 6 (2013) No. 5, p. 67-84.

[4]. Ren Zhouqiao, Chen Jian, Cheng Jieliang, et al. Knowledge-based fertilization recommendation system and application. Transactions of the Chinese Society of Agricultural Engineering. Vol. 27(2011) No. 12, p. 126-133.

[5]. Liu Xuexia. Study on knowledge-based intelligent fault diagnosis of hydraulic system[J]. Telkomnika-Indonesian Journal of Electrical Engineering. Vol. 10 (2012) No. 8, p. 2041-2046. 